\title{
Fluorescent microsphere technique to measure cerebral blood flow in the rat
}

\author{
G De Visscher ${ }^{1}, M_{\text {Haseldonckx }}^{2}$ and W Flameng ${ }^{1}$
}

\begin{abstract}
${ }^{1}$ Laboratory for Experimental Cardiac Surgery, Katholieke Universiteit Leuven, Minderbroedersstraat 17, B-3000 Leuven, Belgium. ${ }^{2}$ ADME/Tox In-vivo, Johnson \& Johnson Pharmaceutical Research and Development, Janssen Pharmaceutica N.V., Turnhoutseweg 30, B-2340 Beerse, Belgium. Correspondence should be addressed to G.D.V. (geofrey.devisscher@med.kuleuven.be).
\end{abstract}

Published online 7 December 2006; doi:10.1038/nprot.2006.332

The present protocol describes a method for parallel measurement of cerebral blood flow (CBF) using fluorescent microspheres and structural assessment of the same material. The method is based on the standard microsphere technique, embolizing capillaries proportional to the blood flow, but requires dissolution of the tissue to retrieve the microspheres. To link the blood flow to the tissue morphology we modified the technique to fluorescent microspheres, which are quantified in cryo- or vibratome sections, allowing structural analysis by, for example, immunohistochemistry or standard histology. The protocol takes $8 \mathrm{~h} 50$ min, without pauses, to complete, but additional flow measurements or specific protocols can increase the time needed.

\section{INTRODUCTION}

Classical methods for measuring regional blood flow cannot be combined with morphological or cellular assessment of the same tissue. Although MRI provides a measure of the distribution of the blood flow in a certain tissue it is a time consuming and very expensive technique because one needs extremely powerful magnets to study small animals. The microsphere technique is still the golden standard in the experimental field of blood flow measurements but suffers from these shortcomings too. First introduced in 1967, the microspheres were labeled with radioactive materials and were used to study blood flow distribution rather than absolute flow $^{1}$. The general concept of blood flow assessment by microspheres is based on the fact that microspheres slightly larger than the capillaries will embolize these vessels in one passage through the body. The number of trapped microspheres in embolized vessels is a direct measure of the blood flowing through that organ; however, the tissue has to be destroyed to recover the microspheres. A major improvement was the introduction in 1968 of arterial reference sampling enabling the calculation of absolute blood flow ${ }^{2}$.

In the late 1980's and beginning of the 1990's, the radiolabeled microspheres were replaced by non-fluorescent ${ }^{3,4}$ or fluorescent dye-filled microspheres ${ }^{5-8}$. In short, the dye-filled microsphere technique consists of digesting the tissue, retrieving the microspheres by filtration and spectrophotometric determination of the dye concentration after dissolving the microspheres ${ }^{9}$. In general this technique has been applied to brain tissue and very often in this type of tissue the link between cerebral blood flow (CBF) and tissue characteristics is crucial. Therefore, we developed this technique for combining several measurements of regional $\mathrm{CBF}(\mathrm{rCBF})$ and immunohistochemistry or histology ${ }^{10}$ of exactly the same tissue. However, spatial resolution is limited as an accurate blood flow measurement requires 400 microspheres to assess the blood flow within a $10 \%$ error margin in the region of interest ${ }^{11}$. The accuracy of this method is also dependent on good mixing and random distribution of the microspheres when they are injected into the aortic branch. In this respect it is important that when CBF is measured, the blood vessels entering and leaving the brain remain untouched. Although it has been shown by means of radiolabeled microspheres that an occluded carotid artery has no effects during normal conditions, it causes serious differential flow distribution in hypercapnia ${ }^{12}$. Accessing the left ventricle using a catheter inserted via the arteria brachialis is a good route of microsphere injection when measuring $\mathrm{CBF}^{13}$ and does not lead to artifactual differential distribution over the hemispheres ${ }^{10}$ in our technique.

The technique can be used in models of neuropathology, such as generalized ${ }^{10}$ or localized ischemic events, where the cerebrovascular component of the pathology is of interest and can easily be used for similar questions in other tissues ${ }^{9,14}$. At present we have already used the fluorescent microsphere CBF assessment combined with post hoc evaluation of neuron morphology on semithin $(2 \mu \mathrm{m})$ sections ${ }^{10}$ and used the sections for immunofluorescent staining (see Fig. 1). These applications allow the linking of local perfusion to changes in immunohistochemically assessed changes in cell markers, such as inflammation, apoptosis, necrosis, etc. Although the previous applications are interesting tools, we foresee that by altering certain steps of the protocol, such as fixation, the sections will be suitable for in situ hybridization and laser capture microtomy with applications such as PCR and microarray gene expression profiling. When using cryosections, the technique has the potential to link perfusion changes to HPLC measured changes in high energy phosphates, such as ATP. Another interesting application, largely depending on the image analysis software used, is 3D mapping of the microspheres in the tissue, which will enable high spatial resolution measurement of blood flow ${ }^{15}$. Although a bolus injection of 48,000 radiolabeled microspheres is sufficient to get 400 microspheres in the brain of a rat and consequently have an accurate CBF measurement ${ }^{13}$, this number of microspheres will not allow the study of regional differences in the rat brain. At least double the amount is necessary to study hemispheric differences in normal conditions, but even more are needed to study ischemic flow patterns ${ }^{10}$. As the underlying mechanism of flow measurement is embolizing small blood vessels this also imposes some limitations. In rats it was shown that a bolus of $10^{6}$ microspheres did not cause hemodynamic effects whereas $2 \times 10^{6}$ microspheres caused a significant decrease in cardiac output $^{16}$. The same study also showed that the total dose of $2 \times 10^{6}$ microspheres was well tolerated if it was given in four 

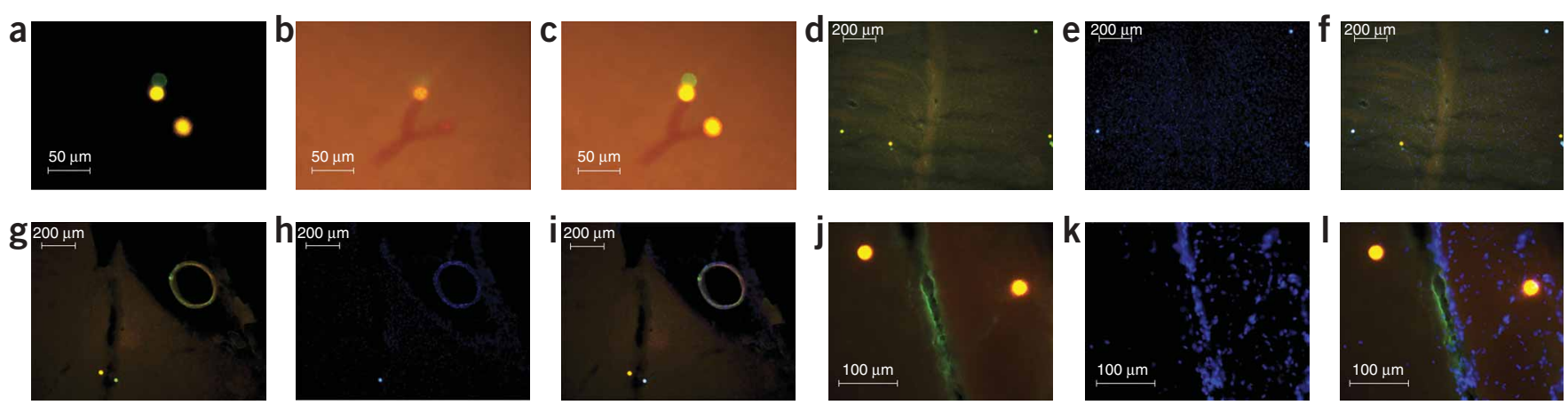

Figure 1 | Micrographs of unstained and immunofluorescent stained brain sections containing white and/or eosin microspheres. (a) Unstained section showing eosin and white microspheres appearing as orange and green, respectively, with a FITC microscopy filter. (b) The same section as in a with light microscopy showing an arterial bifurcation. (c) Merged image of $\mathbf{a}$ and $\mathbf{b}$ showing eosin and white microspheres colocated with the branches of the bifurcation. (d) White and eosin (yellow fluorescent) microspheres in a section stained for alpha smooth muscle actin immunofluorescence. (e) The same region as in $\mathbf{d}$ visualized with a DAPI microscopy filter, showing nuclei and white microspheres in blue fluorescence only. (f) Merged image of $\mathbf{d}$ and $\mathbf{e}$ showing the microsphere distribution. (g) White (green fluorescent) and eosin (yellow fluorescent) microspheres in a section stained for alpha smooth muscle actin immunofluorescence showing a large positive vessel (FITC: green circle). (h) The same region as in $\mathbf{g}$ visualized with a DAPI microscopy filter, showing nuclei and one white microsphere in blue fluorescence. (i) Merged image of $\mathbf{g}$ and $\mathbf{h}$ showing the colocation of the blue stained nuclei and green alpha smooth muscle positive cells. (j) Eosin microspheres next to a smooth muscle heavy chain stained blood vessel (FITC: green). (k) The same region as in $\mathbf{g}$ visualized with a DAPI microscopy filter, showing nuclei from the blood vessel and neighboring tissue (blue). (l) Merged image of $\mathbf{j}$ and $\mathbf{k}$. Scale bars represent $50 \mu \mathrm{m}$ in panels a to $\mathbf{c}$; $200 \mu \mathrm{m}$ in panels $\mathbf{d}$ to $\mathbf{i}$ and $100 \mu \mathrm{m}$ in panels $\mathbf{j}$ to $\mathbf{l}$.

boli of $5 \times 10^{5}$ microspheres. These limitations, in rats, will therefore allow a smaller region of interest representing approximately $10 \%$ of a cerebral hemisphere, or slightly smaller in highly perfused areas. If it is necessary to accurately study smaller regions a larger laboratory animal can be used.

As indicated in the aforementioned sections of the INTRODUCTION, it is not the scope of the current protocol to provide the histology and immunohistochemistry protocols for which the sections are suitable; we have provided an example protocol and recommendations on how to handle the sections. The microspheres used are soluble in organic solvents therefore one has to be cautious as to which products to use. If organic solvents have to be used for certain staining protocols we recommend counting the microspheres first. Certain histological stains (e.g., eosin) or mounting media (e.g., glycerol) are also fluorescent and need to be applied after counting as well. Standard immunofluorescent stains, not using solvents, are perfectly possible and an example of such a protocol is given. The complete protocol is visualized in Fig. 2.

\section{MATERIALS}

\section{REAGENTS}

- Rats (Wistar) ! CAUTION All experiments are to be performed in accordance with relevant authorities' guidelines and regulations.

- Potassium hydroxide (KOH) pellets, purity: $\geq 84 \%$ (Merck, cat. no.

1.05021.5000) ! CAUTION Caustic substance.

- Saline, 0.9\% (w/v; Baxter, cat. no. AKB1322)

- Heparin, 5,000 UI ml ${ }^{-1}$ (Leo Pharma, cat. no. 012866-04) $\triangle$ CRITICAL If another concentration of heparin is used ensure that the injected dose is the same.

- Paraformaldehyde, extra pure (Merck, cat. no. 1.04005.1000) ! CAUTION

Harmful by inhalation and if swallowed, irritating to eyes, skin and respiratory system.

- Tween 80 (Sigma, cat. no. P1754)

- Aquamount (BDH Laboratory Supplies) $\triangle$ CRITICAL If a mounting solution containing organic solvents is used the microspheres will dissolve.

- Poly-L-lysine solution (Sigma-Aldrich, cat. no. 9820)

- Tissue freezing medium (OCT Cryocompound; Leica, cat. no. 020108926 )

-2-methylbutane (Sigma, cat. no. 270342)

- Liquid nitrogen ! CAUTION Liquid at $-196{ }^{\circ} \mathrm{C}$, handle carefully

- Thimerosal, sodium ethylmercurythiosalicylate (Fluka, cat. no. 71230)

- Phosphate buffered saline (PBS) tablets (Sigma, cat. no. P4417-50TAB)

- Sucrose (Calbiochem, cat. no. 5737)

- Triton X 100 (Sigma, cat. no. 93443)

- Bovine serum albumin (BSA; Sigma, cat. no. A3294-50G)

- Goat pre-immune serum (Dako, cat. no. X0907)

- Mouse anti-human anti-alpha smooth muscle antibody (clone: 1A4; Dako, cat. no. M0851)
- Mouse anti-human anti-smooth muscle heavy chain myosin (clone: G-4;

Dako, cat. no. M3558)

- FITC labeled goat anti-mouse antibody (Dako, cat. no. F0479)

- Vectashield (Vector Laboratories Inc., cat. no. H-1500)

- Xylocaine 2\% (w/v, Lidocain hydrochlorid; AstraZeneca, cat. no. 0137-562)

\section{EQUIPMENT}

- Small animal anesthesia setup

- Small animal endotracheal intubation tube (homemade, see EQUIPMENT SETUP)

- Tape

- Surgical tools

- Suture; 3-0 Surgilon precut lengths (American Cyanamid Co., cat. no. 1918-41)

- Two nerve and tendon hooks (Medicon, cat. no. 20.22.01)

- Arterial microvascular clamp by Acland (S\&T, cat. no. 00401)

- PE10 tubing (Becton Dickinson, cat. no. 427401)

- PE50 tubing (Becton Dickinson, cat. no. 427411)

- PE205 tubing (Becton Dickinson, cat. no. 427445)

- Cyanoacrylate glue (Bison Super Glue Industrial)

- Heating pad with rectal temperature feedback (homemade but Harvard

Apparatus provides comparable products)

- Hypodermic needles 26G (Becton Dickinson, cat. no. 303800)

- Hypodermic needles 18G (Becton Dickinson, cat. no. 301900)

- Hypodermic needles 16G (Becton Dickinson, cat. no. 300637)

- Syringes, $1 \mathrm{ml}$ (Plastipac; Becton Dickinson, cat. no. 300013)

- Perfusor syringe OPS, $50 \mathrm{ml}$ (B.Braun, cat. no. 8728844F)

- Blood pressure monitor system (homemade but Harvard Apparatus provides comparable products) 
- Glass syringe, $10 \mathrm{ml}$ with Luer tip (VWR, cat. no. 612-0315)

-Withdrawal/infusion pump (Harvard Apparatus)

- Fluorescent polystyrene microspheres, $15 \mu \mathrm{m}$ (Fluospheres; Invitrogen, yellow-green fluorescence cat. no. F8842 and red fluorescence cat. no. F8844) (Dye-Trak microspheres; Triton Technology, white cat. no. 155-0370 and eosin cat. no. 155-0531)

- Sonicator (VWR, cat. no. 142-6045)

- Vortex mixer type VV3 (VWR, cat. no. 444-0007)

- Hamilton syringe, $250 \mu$ with Luer tip (VWR, cat. no. 1725 LT)

- Tubes with screw caps (Sarstedt, cat. no. 60541004)

- Disposable pressure infusor (Statcorp, cat. no. 803S)

-3-way tap (Connecta plus 3; Becton Dickinson, cat. no. 394600)

- Infusor set (Codan Medizinische Geräte GmbH, cat. no. 16/43.3977)

- Water bath

- Polyester filter membranes (Dye-Trak, $25 \mathrm{~mm}$ diameter and $10 \mu \mathrm{m}$ pore size;

Triton Technology, cat. no. 31079)

-Water jet vacuum pump (VWR, cat. no. 181-9100)

- Aspiration Erlenmeyer Schott (VWR, cat. no. 511-0326)

- Conical rubber plug (VWR, cat. no. 217-0217)

-Whatman Borosilicate filter holders, 25 mm diameter (VWR, cat. no. 511-0137)

- Microscope slides, Superfrost (Menzel-Gläser)

- Cover slip, $24 \times 32 \mathrm{~mm}$ (Menzel-Gläser)

- Vibratome (Leica) or cryostat (Microm)

- Cryomolds (Bayer NV, cat. no. 05583045)

- Fluorescence microscope with image analysis system (Axioplan 2 imaging

microscope with Axiovision 4.4 software; Zeiss)

REAGENT SETUP

KOH solution For the digestion of the control samples a $16 \mathrm{~N} \mathrm{KOH}$ solution is prepared by dissolving $89.8 \mathrm{~g}$ of $\mathrm{KOH}$ pellets in $100 \mathrm{ml}$ distilled water. Allow the solution to cool down and add $0.5 \%(\mathrm{v} / \mathrm{v})$ Tween 80 ! CAUTION Since this is a caustic substance generating excessive heat when dissolving in water, one

should wear gloves, safety goggles and a lab coat when preparing the solution.

\section{PROCEDURE}

\section{Animal preparation $\bigcirc$ TIMING $\mathbf{4 0}$ min}

1| Induce anesthesia, intubate the animal and connect the endotracheal tube to the breathing circuit of the anesthesia setup. Place the animal in a dorsal position on a heating pad.

$\triangle$ CRITICAL STEP The type of anesthesia used can potentially influence the results by their effect on the CBF.

\section{2| Cannulate the left femoral artery with the PE50 cannula} as follows: remove a patch of skin next to the groin and bluntly dissect the fat tissue until both femoral vein and artery are visible. Loosen the vein and artery from the surrounding tissue and each other, using the nerve hooks. Ligate the distal end of the artery with the precut sutures and prepare the proximal knot but do not tie it completely. Fill the catheter with heparin solution using a 1-ml syringe and leave it attached to the syringe. Clamp the artery and make a small incision through which the catheter is inserted. Remove the clamp, tie the proximal knot and tie the ends of the distal ligation around the cannula.

3| Place the microsphere injection catheter as follows: place the animal in a dorsal position with its right side towards you and fix the left paw perpendicular to the body using a piece of tape. Fix the right paw at approximately a $20^{\circ}$ angle to the head, again using some tape. Remove a patch of skin in the armpit and bluntly dissect the fat tissue until the arteria brachialis is visible (Fig. 3). Gently remove the artery from the surrounding tissue, ligate the distal end and prepare the proximal knot. Fill the catheter with heparin solution using a 1-ml
Paraformaldehyde solution For fixation of the brain a $4 \%(\mathrm{w} / \mathrm{v})$ paraformaldehyde solution is prepared. $10 \mathrm{~g}$ paraformaldehyde is added to $250 \mathrm{ml}$ saline, this is gently heated just below boiling point until the paraformaldehyde is dissolved. The solution is then placed on ice and used ice-cold only.

$\Delta$ CRITICAL This solution can only be used for $24 \mathrm{~h}$ after it has been prepared. ! CAUTION Harmful by inhalation and if swallowed, irritating to eyes, skin and respiratory system.

Heparin solution To prevent blockage of the catheters they are filled with a $50 \mathrm{IU} \mathrm{ml}^{-1}$ heparin in saline solution. The solution is made by injection of $2.5 \mathrm{ml}$ of the heparin in a commercially available $250 \mathrm{ml} 0.9 \%$ (w/v) saline container Phosphate buffered saline (PBS) Dissolve one tablet in $200 \mathrm{ml}$ of deionized water. This solution serves as a basis for the immunoassay solutions and sample storage.

\section{EQUIPMENT SETUP}

Cannulae Two cannulae need to be prepared. For arterial blood sampling and blood pressure monitoring a $26 \mathrm{G}$ needle is inserted in one end of approximately $30 \mathrm{~cm}$ PE50 tubing. This is then fixed with a drop of glue. The second cannula, which will be used for microsphere injection, is basically the same but at the other end a $10 \mathrm{~cm}$ length of PE10 tubing is inserted (approximately $15 \mathrm{~mm}$ ) and fixed with glue.

Endotracheal tube The endotracheal tube is made by shortening the 16G needle to approximately $10 \mathrm{~mm}$ and inserting this in $5 \mathrm{~cm}$ of PE205 tubing. Perfusion system Build a perfusion system by connecting an empty saline bag to the infusor set and closing the other side with a 3-way tap. Attach a blunted $18 \mathrm{G}$ hypodermic needle to the other end of the 3-way tap. Insert the empty saline bag in the deflated disposable pressure infusor.

Coated slides Dilute the poly-L-lysine solution 10 times with deionized water. Place the clean microscope slides in this solution for $5 \mathrm{~min}$ and let the slides dry Negative pressure filter system Drill a hole with the appropriate diameter in the conical rubber plug and insert the bottom part of the filter holder. Connect the water jet pump to a water tap and to the aspiration bottle. Insert the conical plug in the top opening of the aspiration bottle.

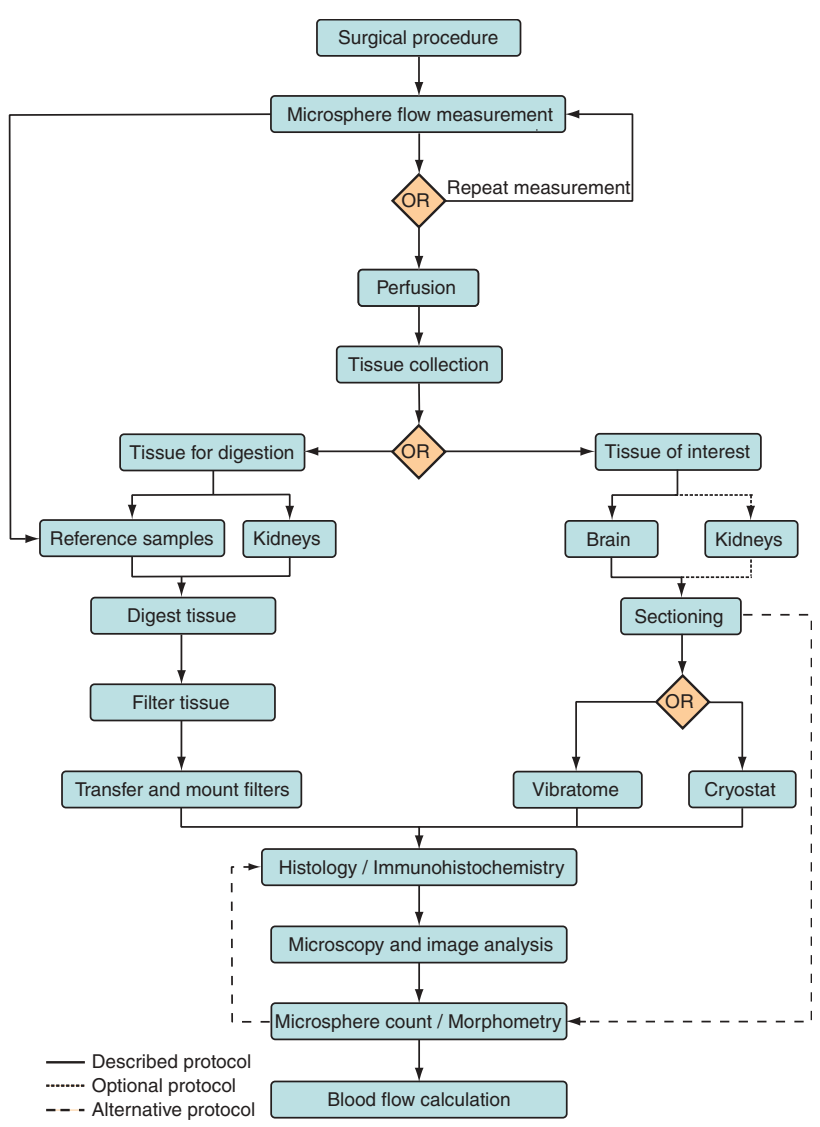

Figure 2 | Flow chart of the protocol. Blue fields represent protocol steps, pink fields indicate different options. 
syringe and leave it attached to the syringe. Estimate the distance from the arteria brachialis to the heart and mark the distance on the cannula. Make a small incision in the artery and insert the cannula. Attach the cannula to the blood pressure monitoring device. Gently advance the cannula towards the heart. At approximately the mark on the cannula a sudden drop in diastolic blood pressure will occur, at that point the cannula is in the left ventricle. Advance the cannula another $2 \mathrm{~mm}$, then tie the proximal knot and tie the ends of the distal ligation around the cannula. Disconnect the cannula from the blood pressure monitor and attach the cannula to the syringe.

$\triangle$ CRITICAL STEP The arteria brachialis is a very small blood vessel which needs to be handled with care because it can easily be ruptured or go into vasoconstriction during the described procedure. It is necessary to have this cannula in the left ventricle to obtain valid data.

? TROUBLESHOOTING

4| Place the animal in the upright position and verify the position of the microsphere injection cannula by checking the diastolic blood pressure.

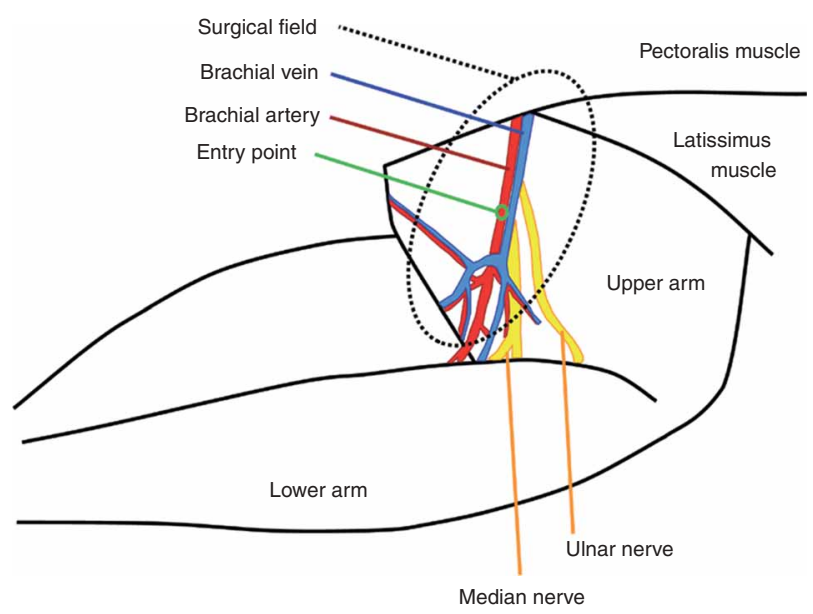

Figure 3 | Diagram of the surgery. Arteries are represented in red, veins in blue and nerves in yellow. After ligation of the distal (towards lower arm) and clamping proximal (towards pectoral muscle) part of the brachial artery, a small incision is made at the entry point mark.

\section{Microsphere infusion TIMING 10 min}

5| Sonicate the microspheres for $5 \mathrm{~min}$ at room temperature $\left(20-25^{\circ} \mathrm{C}\right)$.

6| Meanwhile, aspire $0.5 \mathrm{ml}$ of undiluted heparin in the glass syringe and mount it in the withdrawal pump. Connect the arterial blood cannula (arteria femoralis) to the syringe.

7| Vortex the microspheres for $30 \mathrm{~s}$ at room temperature and aspire the required amount of microspheres into the Hamilton syringe.

$\triangle$ CRITICAL STEP The amount of microspheres will determine the volume of the region of interest since 400 microspheres are required per region of interest. We used 100,000 microspheres for each measurement, which is enough to study the different hemispheres. Since different regions of interest might be used, we recommend performing a pilot study to determine the amount of microspheres one should use for the particular region of interest one wants to assess.

8| Start the withdrawal pump prior to the microsphere injection and ensure the blood is entering the syringe.

$\triangle$ CRITICAL STEP The withdrawal rate should be chosen according to the amount of microspheres injected. The more microspheres used the lower the withdrawal rate can be, but lower withdrawal rates can increase variability. We used $0.566 \mathrm{ml} \mathrm{min}^{-1}$.

? TROUBLESHOOTING

9| Connect the Hamilton syringe to the microsphere injection cannula and hold it in a vertical position while slowly injecting the microspheres over approximately $20 \mathrm{~s}$. Replace the Hamilton syringe with a 1-ml syringe filled with heparin solution and flush the cannula for $20 \mathrm{~s}$ with $0.5 \mathrm{ml}$.

10| Switch off the withdrawal pump after 2 min during which the previous step was finalized.

11 Transfer the blood sample to a plastic tube and rinse the syringe two times with $2 \mathrm{ml}$ heparin solution. Add $1 \mathrm{ml}$ of the $16 \mathrm{~N} \mathrm{KOH}$ solution, cap the tube and digest overnight at $78{ }^{\circ} \mathrm{C}$ in the water bath.

12| Repeat the whole process with another color of microsphere for additional blood flow measurements.

\section{Transcardial perfusion and tissue collection $\bigcirc$ TIMING $\mathbf{3 0}$ min}

13| Fill the perfusion system via the 3-way tap with $200 \mathrm{ml}$ of ice cold paraformaldehyde solution using the 50-ml syringe and de-air the system. Inflate the pressure infusor until the pressure gauge reads $200 \mathrm{~mm} \mathrm{Hg}$.

14 Put the rat in a dorsal position in a tray, open the thorax and lift the heart with forceps. Inject $0.5 \mathrm{ml}$ of undiluted heparin in the left ventricle. Remove the endotracheal tube. Make a small incision and insert the $18 \mathrm{G}$ blunted needle of the perfusion system in the left ventricle and advance it through the aortic valve into the aortic root (Fig. 4). 
! CAUTION Although the described process does not require supplemental anesthesia or killing the animal, the local ethics committee or laws might require one of these processes.

15| Fix the needle with a clamp, open the 3-way tap and cut the right atrium. Keep the pressure infusor pressurized and perfuse the rat until the bag is empty.

$\triangle$ CRITICAL STEP Ensure that no air bubbles are being infused since they can embolize the arteries leading to un-perfused areas.

\section{? TROUBLESHOOTING}

16| Decapitate the rat and remove excess tissue but leave the skull intact. Post-fix the skull in the remaining paraformaldehyde for $24 \mathrm{~h}$ at $4^{\circ} \mathrm{C}$.

$\triangle$ CRITICAL STEP Removing the brain at this point might alter its dimension, especially in badly perfused areas such as infarct zones. handle in one of two ways: (A) post-fixation or (B) $\mathrm{KOH}$ digestion.

\section{(A) Post-fixation}

(i) Post-fix the kidneys for $24 \mathrm{~h}$ together with the head. tissue)

\section{(B) $\mathrm{KOH}$ digestion} distilled water. Filtering the digested tissue). PBS with a few crystals of Thimerosal.

\section{Sectioning of the tissue TIMING $\mathbf{1}$ h $\mathbf{3 0}$ min}

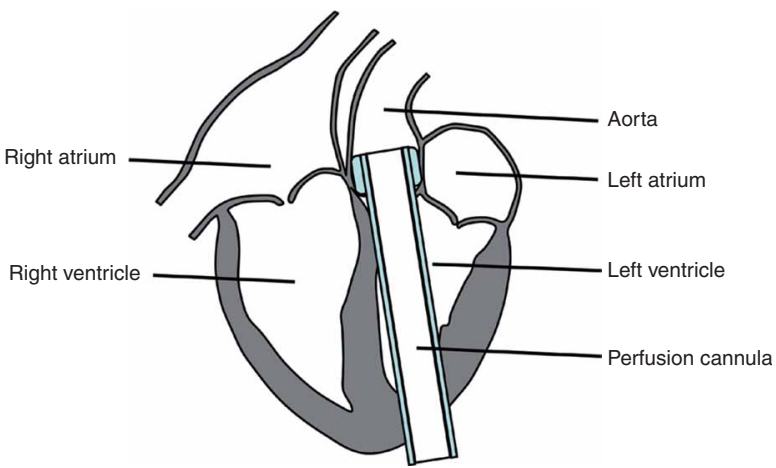

Figure 4 | Diagram of the perfusion cannula placement. After opening the thorax and pericardium a small incision at the apex is made. The blunt cannula is entered through the opening and gently inserted in through the aortic valve into the aorta. The rat aorta is translucent so the cannula is clearly visible once in place. The cannula is clamped in place and the perfusion can be started.

17| Remove the kidneys, which serve as an internal control for adequate microsphere mixing and distribution in the blood, and

(ii) Process the kidneys further in the same way as the brain (for further information see the section on Sectioning of the

(i) Weigh and digest the kidneys overnight by inserting them in the tubes and adding $2.5 \mathrm{ml} 16 \mathrm{~N} \mathrm{KOH}$ solution and $7.5 \mathrm{ml}$

(ii) To speed up the digestion place the tubes overnight in the water bath at $78^{\circ} \mathrm{C}$ (for further information see the section on

PAUSE POINT The samples can be kept for several days before further processing, but keep the fixed tissues at $4{ }^{\circ} \mathrm{C}$ in

18| Carefully remove the brain from the skull (after Step 16) prior to sectioning. Isolate the region of interest by cutting away excess tissue.

19| For sectioning of the brain and kidneys two options are available: (A) vibratome sectioning or (B) cryo-sectioning.

\section{(A) Sectioning brain with a vibratome}

(i) Fix the brain or kidney sample to the tissue holder with the cyanoacrylate glue.

(ii) Trim the sample until complete sections are obtained. Discard the first complete section since it will not be of constant thickness.

(iii) Put each subsequent section on a separate coated slide and let them air dry.

$\triangle$ CRITICAL STEP The thickness of the section needs to be constant and largely exceeding the diameter of the microspheres used. We used $100 \mu \mathrm{m}$ sections.

- PAUSE POINT The vibratome sections can be stored in a dry space for a few weeks at room temperature.

\section{(B) Sectioning brain with a cryostat}

(i) Incubate the samples overnight in $20 \%(\mathrm{w} / \mathrm{v})$ sucrose.

(ii) Place the samples in the cryomolds pre-filled with tissue freezing medium and place them into a beaker with cold 2-methylbutane to freeze immediately in liquid nitrogen.

PAUSE POINT The frozen blocks can be stored at $-80{ }^{\circ} \mathrm{C}$ for years.

\section{? TROUBLESHOOTING}

(iii) Fix the samples to the pre-cooled tissue holder with a drop of tissue freezing medium and allow equilibration of the samples so the whole system is $-20^{\circ} \mathrm{C}$.

(iv) Trim the sample until complete sections are obtained. Discard the first complete section as it will not be of constant thickness. 
(v) Put each subsequent section on a separate coated slide and store them at $-20^{\circ} \mathrm{C}$.

$\triangle$ CRITICAL STEP The thickness of the sections needs to be constant and largely exceeding the diameter of the microspheres used. We used $100 \mu \mathrm{m}$ sections.

PAUSE POINT The cryosections can be kept at $-20{ }^{\circ} \mathrm{C}$ for several months.

Filtering the digested tissue TIMING 30 min (per sample obtained in Step 17B)

20| Insert a polyester filter in the filter holder of the negative pressure system. Vortex the dissolved tissue (kidney) and gently pour over the filter. Refill the tube with $\mathrm{KOH}$ solution, pour it over the filter and repeat two more times.

? TROUBLESHOOTING

21 Carefully remove the filter and place it on a microscope slide.

$\triangle$ CRITICAL STEP Do not allow the filter to dry completely as this can cause loss of microspheres.

22| Mount the slide with two drops of aquamount and a cover slip. Insert a new filter in the filter holder and reapply the filtrate over the same filter holder.

23| Wash the material and filter the washing solution, repeat Steps 21, 22 and 23 until three mounted filters for each tissue sample are obtained.

$\triangle$ CRITICAL STEP Previous work has shown that this repetition is necessary to obtain approximately $97 \%$ of the microspheres in the sample ${ }^{10}$.

PAUSE POINT The mounted filter can be kept for a few weeks at room temperature before analysis.

Immunohistochemistry and/or histology $\odot \operatorname{TIMING} \mathbf{4} \mathbf{h}$

24| Rinse the slides three times for 5 min with PBS containing $0.1 \%(v / v)$ Triton $X 100$ detergent.

25 Incubate the slides for 45 min in PBS containing $0.1 \%(v / v)$ Triton X 100 detergent, $2 \%(w / v)$ BSA and 10\% (v/v) goat pre-immune serum at room temperature.

26 Incubate the slides for $1 \mathrm{~h}$ in PBS containing $0.1 \%(\mathrm{v} / \mathrm{v})$ Triton $\mathrm{X} 100$ detergent, $2 \%(\mathrm{w} / \mathrm{v})$ BSA and 1\% (v/v) mouse anti-human anti-alpha smooth muscle antibody (clone: 1A4) or anti-smooth muscle heavy chain myosin (clone; G-4) at room temperature.

27| Wash the slides three times for 5 min with PBS containing $0.1 \%(\mathrm{v} / \mathrm{v})$ Triton X 100 detergent.

28| Incubate the slides for 45 min in PBS containing $0.1 \%(v / v)$ Triton X 100 detergent, 2\% (w/v) BSA and 0.5\% (v/v) FITC labeled goat anti-mouse antibody at room temperature. From hereon the slides have to remain shielded from light.

29| Wash the slides three times for 5 min with PBS containing $0.1 \%(\mathrm{v} / \mathrm{v})$ Triton $X 100$ detergent.

30| Mount with Vectashield containing DAPI to visualize the nuclei (for examples see Fig. 1d-l).

\section{CBF calculation TIMING 1 h 30 min}

31| Count the microspheres in the kidney slices or those on filters derived from digested kidney using a fluorescence microscope. This can be done by hand or, if the image analysis system allows, automatically.

32| Measure the surface of the counted kidney sections by means of the microscope and image analysis system. The image analysis system has to be calibrated beforehand to know the pixel size. For this a calibration grid can be used but the further process is dependent on the used image analysis software.

33| Calculate the concentration of microspheres in the kidneys by dividing the number of microspheres by either the total surface (for kidney sections) or the weight (for digested kidneys).

$\triangle$ CRITICAL STEP If a difference in microsphere concentration between the left and right kidney exceeding $10 \%$ is found, this indicates that the microspheres were not mixed very well in the blood and that the experiment should be excluded because of artificial differences in blood flow distribution.

? TROUBLESHOOTING

34| Count the microspheres on the filters of the blood sample.

35| Count the microspheres in, and measure the surface of, the brain sections by means of the microscope and image analysis system. 
PROTOCOL

36 Calculate the measured brain volume, for practical reasons in $\mathrm{mm}^{3}$, by adding up the measured surfaces and multiplying them with the thickness of the sections (100 $\mu \mathrm{m}$ in our case).

37| Calculate the blood flow with the following formula:

$$
\mathrm{Q}=((\mathrm{FR} \times \mathrm{CT}) /(\mathrm{CR} \times \mathrm{VT})) \times 10^{5}
$$

Where $Q$ is flow in $\mathrm{ml} \mathrm{min} \mathrm{m}^{-1} 100 \mathrm{~cm}^{-3}$; FR is flow rate of the reference sample $\left(\mathrm{ml} \mathrm{min}{ }^{-1}\right)$; $C R$ is the microsphere count in the reference sample; $\mathrm{CT}$ is the microsphere count in the tissue; and VT is the tissue volume $\left(\mathrm{mm}^{3}\right)$.

\section{? TROUBLESHOOTING}

\section{TIMING}

Steps 1-4, Animal preparation: $40 \mathrm{~min}$

Steps 5-12, Microsphere infusion: $10 \mathrm{~min}$

Steps 13-17, Transcardial perfusion and tissue collection: $30 \mathrm{~min}$

Steps 18-19, Sectioning of the tissue: $1 \mathrm{~h} 30 \mathrm{~min}$

Steps 20-23, Filtering the digested tissue: 30 min (per sample from Step 17B)

Steps 24-30, Immunohistochemistry and/or histology: $4 \mathrm{~h}$

Steps 31-37, CBF calculation: 1 h 30 min

\section{? TROUBLESHOOTING}

Troubleshooting advice can be found in Table 1.

TABLE 1 | Troubleshooting table.

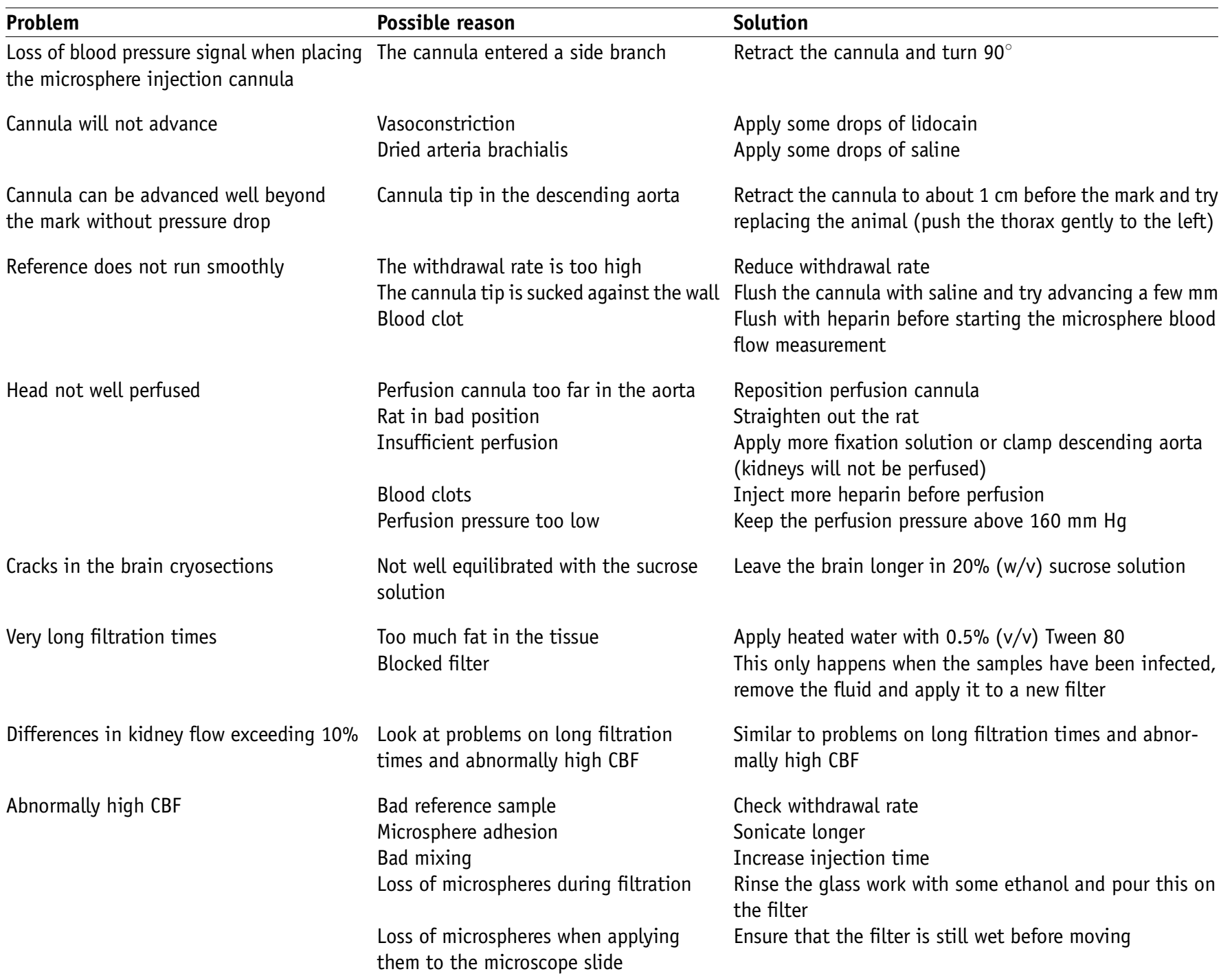


PROTOCOL

TABLE 2 | Example of raw section microsphere counts.

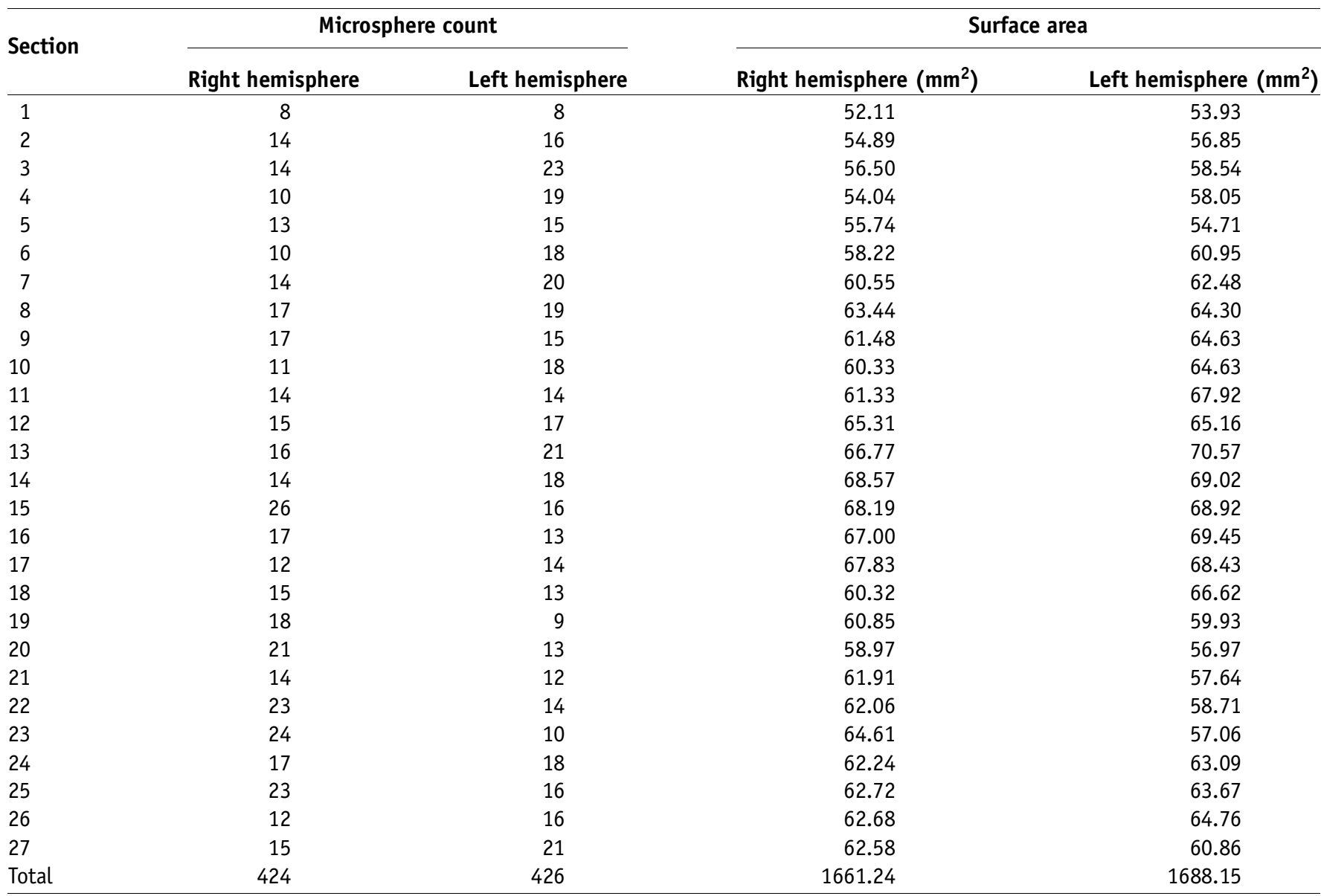

\section{ANTICIPATED RESULTS}

The overall anticipated result is to obtain a rCBF measurement of tissue (e.g., brain) by applying the "gold standard" of blood flow measurement to tissue that can then still be used for other analyses. To illustrate the results a whole calculation of hemispheric blood flow during hypercapnia from one animal is given. The raw data obtained from counting the microspheres in the sections, as well as measurement of the section surfaces, is given in Table 2. The total data, listed in the last row, are than used in the calculation of the hemispheric blood flows. In this particular experiment, a 0.566 reference sample flow rate was used, the sections were $100 \mu \mathrm{m}$ thick and the reference sample contained 336 microspheres. Using the described formula we obtained the following flow for the right hemisphere:

$$
\mathrm{Q}=((\mathrm{FR} \times \mathrm{CT}) /(\mathrm{CR} \times \mathrm{VT})) \times 10^{5}
$$

Where $Q$ is flow in $\mathrm{ml} \mathrm{min}^{-1} 100 \mathrm{~cm}^{-3}$

FR is flow rate of the reference sample $\left(\mathrm{ml} \mathrm{min}^{-1}\right): 0.566 \mathrm{ml} \mathrm{min}^{-1}$

CR is the microsphere count in reference sample: 336 microspheres

$\mathrm{CT}$ is the microsphere count in the tissue: 424 microspheres

VT is the tissue volume $\left(\mathrm{mm}^{3}\right): 1661.24 \times 0.1=166.12$

$$
\mathrm{Q}=((0.566 \times 424) /(336 \times 166.12)) \times 10^{5}=429 \mathrm{ml} \mathrm{min}^{-1} \text { per } 100 \mathrm{~g} \text { weight }
$$

For the left hemisphere the following was obtained:

$$
\mathrm{Q}=((0.566 \times 426) /(336 \times 168.82)) \times 10^{5}=425 \mathrm{ml} \mathrm{min}^{-1} \text { per } 100 \mathrm{~g} \text { weight }
$$

The CBF measurement technique described enables one to study the effects of perfusion changes on cellular, molecular or biochemical changes or vice versa. By itself the technique allows accurate measurement of the blood flow as all the microspheres present in the tissue can clearly be visualized in unstained (Fig. 1a-c) or immunofluorescent stained sections (Fig. 1d-f). Combined with other techniques, such as immunofluorescent staining, the technique gives additional information. Here we have 
PROTOCOL

shown with antibodies against alpha smooth muscle actin (Fig. $\mathbf{1 g - i}$ ) and smooth muscle heavy chain myosin (Fig. $\mathbf{1 j - l}$ ) that the microspheres reside in blood vessels that do not have a smooth muscle layer, i.e., capillaries.

COMPETING INTERESTS STATEMENT The authors declare that they have no competing financial interests.

Published online at http://www.natureprotocols.com

Reprints and permissions information is available online at http://npg.nature.com/ reprintsandpermissions

1. Rudolph, A.M. \& Heymann, M.A. The circulation of the fetus in utero. Methods for studying distribution of blood flow, cardiac output and organ blood flow. Circ. Res. 21, 163-184 (1967).

2. Makowski, E.L., Meschia, G., Droegemueller, W. \& Battaglia, F.C. Measurement of umbilical arterial blood flow to the sheep placenta and fetus in utero. Distribution to cotyledons and the intercotyledonary chorion. Circ. Res. 23, 623-631 (1968).

3. Kowallik, P. et al. Measurement of regional myocardial blood flow with multiple colored microspheres. Circulation 83, 974-982 (1991).

4. Hale, S.L., Alker, K.J. \& Kloner, R.A. Evaluation of nonradioactive, colored microspheres for measurement of regional myocardial blood flow in dogs. Circulation 78, 428-434 (1988).

5. Glenny, R.W., Bernard, S. \& Brinkley, M. Validation of fluorescent-labeled microspheres for measurement of regional organ perfusion. J. Appl. Physiol. 74, 2585-2597 (1993).

6. Austin, G.E. et al. Determination of regional myocardial blood flow using fluorescent microspheres. Am. J. Cardiovasc. Pathol. 4, 352-357 (1993).

7. Mori, H. et al. New nonradioactive microspheres and more sensitive X-ray fluorescence to measure regional blood flow. Am. J. Physiol. 263, H1946-H1957 (1992).
8. Morita, Y. et al. Local blood flow measured by fluorescence excitation of nonradioactive microspheres. Am. J. Physiol. 258, H1573-H1584 (1990).

9. Fluorescent Microsphere Resource Center. Microsphere manual http://fmrc.pulmcc.washington.edu/2006.

10. De Visscher, G. et al. Development of a novel fluorescent microsphere technique to combine serial cerebral blood flow measurements with histology in the rat. J. Neurosci. Methods 122, 149-156 (2003).

11. Buckberg, G.D. et al. Some sources of error in measuring regional blood flow with radioactive microspheres. J. Appl. Physiol. 31, 598-604 (1971).

12. De Ley, G., Nshimyumuremyi, J.B. \& Leusen, I. Hemispheric blood flow in the rat after unilateral common carotid occlusion: evolution with time. Stroke 16, 69-73 (1985)

13. Nakai, M., Tamaki, K., Yamamoto, J., Shimouchi, A. \& Maeda, M. A minimally invasive technique for multiple measurement of regional blood flow of the rat brain using radiolabeled microspheres. Brain Res. 507, 168-171 (1990).

14. Luchtel, D.L., Boykin, J.C., Bernard, S.L. \& Glenny, R.W. Histological methods to determine blood flow distribution with fluorescent microspheres. Biotech. Histochem. 73, 291-309 (1998).

15. Bernard, S.L. et al. High spatial resolution measurements of organ blood flow in small laboratory animals. Am. J. Physiol. Heart Circ. Physiol. 279, H2043-H2052 (2000).

16. Kobayashi, N., Kobayashi, K., Kouno, K., Horinaka, S. \& Yagi, S. Effects of intraatrial injection of colored microspheres on systemic hemodynamics and regional blood flow in rats. Am. J. Physiol. 266, H1910-H1917 (1994). 